\title{
Relação do uso do solo com a diversidade e a atividade da fauna edáfica
}

\author{
Marciane Danniela Fleck PESSOTTO ${ }^{1}$, Natielo Almeida SANTANA ${ }^{1}$, Rodrigo Josemar Seminoti JACQUES ${ }^{1}$, \\ Joice Aline FREIBERG ${ }^{*}$, Dayanna do Nascimento MACHADO ${ }^{1}$, Eliara Marin PIAZZA, \\ Lethícia ROSA NETO ${ }^{1}$, Zaida Ines ANTONIOLLI
}

${ }^{1}$ Centro de Ciências Rurais, Universidade Federal de Santa Maria, Santa Maria, RS, Brasil. (ORCID: 0000-0001-7783-7907; 0000-0003-2461-144X; 0000-0002-8240-5145; *; 0000-0001-9837-5369; 0000-0001-6661-074X; 0000-0002-2907-6792; 0000-0003-2036-8710)

*E-mail: jaf.freiberg@gmail.com (ORCID: 0000-0001-9086-9882)

Recebido em 05/02/2020; Aceito em 27/04/2020; Publicado em 22/05/2020.

\begin{abstract}
RESUMO: Objetivou-se avaliar o efeito de diferentes usos do solo na abundância, riqueza, diversidade e atividade de organismos da fauna edáfica. A fauna do solo foi amostrada em: florestamento de Eucalyptus spp., florestamento de Pinus spp., lavoura de grãos, solo impactado pela construção civil e pastagem natural. Para a avaliação dos organismos da fauna epiedáfica empregou-se o método da armadilha de queda e para a amostragem dos organismos da fauna hemiedáfica utilizou-se o método TSBF. A atividade biológica do solo foi avaliada pelo método de lâminas bait. Foram coletados 5.413 organismos epiedáficos e 813 organismos hemiedáficos, distribuídos em 18 grupos taxonômicos. Os florestamentos de Pinus e Eucalyptus abrigaram as maiores abundâncias de organismos epiedáficos, com dominância de Collembola, o que resultou em menor índice de diversidade. O solo afetado pela construção civil apresentou as menores abundância e riqueza de organismos epiedáficos. A abundância, riqueza e diversidade de organismos hemiedáficos foram maiores na pastagem natural e menores no florestamento de Pinus e no solo impactado pela construção civil. A atividade biológica do solo foi maior no florestamento de Eucalyptus e menor no solo impactado pela construção civil. A abundância, riqueza e diversidade da fauna edáfica é afetada pelo uso do solo.

Palavras-chave: atividade biológica do solo; bioindicadores; degradação do solo; invertebrados do solo; qualidade do solo;
\end{abstract}

\section{Relation of soil use with diversity and activity of edaphic fauna}

\begin{abstract}
This study evaluated the effect of different land uses on the abundance, richness, diversity and activity of edaphic fauna. The soil fauna was sampled in: Eucalyptus spp. afforestation, Pinus spp. afforestation, grain cropping, soil impacted by construction and natural grassland. The epiedaphic fauna was sampled by pitfalls and the hemiedaphic fauna by TSBF method. The soil biological activity was evaluated by the laminabait test. 5,413 epiedaphic organisms and 813 hemiedaphic organisms were collected, classified in 18 taxonomic groups. The afforestation of Pinus and Eucalyptus had the greatest abundance of epiedaphic organisms, with dominance of springtails, which resulted in a lower index of diversity. The soil affected by the construction had the lowest abundances and the richness of epiedaphic organisms. The abundance, richness and diversity of hemiedaphic organisms were higher in natural grassland; and smaller in Pinus afforestation and soil impacted by construction. Soil biological activity was higher in the Eucalyptus afforestation and lower in the soil impacted by construction. Soil use affects the abundance, richness and diversity of edaphic fauna.
\end{abstract}

Keywords: soil biological activity; bioindicators; soil degradation; soil invertebrates; soil quality.

\section{INTRODUÇÃO}

A fauna do solo refere-se à comunidade de invertebrados que vivem permanentemente ou que passam um ou mais ciclos de vida no solo. Esta habita diferentes camadas do perfil do solo, sendo que os organismos que vivem na superfície do solo (gafanhotos, aranhas, centopeias, etc) são denominados epiedáficos; os que habitam o horizonte A (algumas espécies de minhocas, larvas de coleópteros, nematoides, etc) são denominados hemiedáficos; e os que vivem nas camadas mais profundas (algumas espécies de minhocas, cupins, formigas, etc) são classificados como euedáficos (GISIN, 1943).

A atividade dos organismos do solo resulta em melhorias das propriedades químicas, físicas e biológicas no solo (MENTA, 2012; CULLINEY, 2013). A fauna edáfica desempenha importantes funções ecossistêmicas pois atua nos processos de decomposição e incorporação dos resíduos orgânicos no solo, mineralização de nutrientes, aumento do teor de matéria orgânica, agregação das partículas, formação de bioporos e controle biológico (BROWN et al., 2015). Ao mesmo tempo em que a fauna edáfica promove melhorias nas propriedades do solo ela também é influenciada pelas alterações destas propriedades, ocasionadas pelo uso e manejo do solo (FERREIRA et al., 2019). Por responder rapidamente às práticas de uso e manejo do solo (CASARIL et al., 2019), a fauna edáfica destaca-se como indicadora da 
qualidade ambiental (ALMEIDA et al., 2016). A perda da diversidade e a alteração da estrutura da fauna edáfica são indicativos da degradação do solo e da perda de sua sustentabilidade, pois os processos acima citados deixam de ocorrer ou ocorrerão em uma menor magnitude (SILVA et al., 2012). Deste modo, objetivou-se avaliar o efeito de diferentes usos do solo na abundância, riqueza, diversidade e atividade de organismos da fauna edáfica.

\section{MATERIAL E MÉTODOS}

O estudo foi realizado no município de Santa Maria, Rio Grande do Sul, Brasil. Os usos do solo avaliados foram: 1) florestamento de Eucalyptus spp. com regeneração no subbosque de espécies nativas da região (FE); 2) florestamento de Pinus spp. com dossel das árvores fechado, havendo pouca incidência direta de luminosidade natural (FP); 3) área destinada para lavoura de grãos mas no momento do estudo em pousio e apresentando espécies espontâneas (LA); 4) solo impactado pela construção civil, compactado e com pouca vegetação de gramíneas (CC); e 5) pastagem natural com vegetação formada por espécies herbáceas de estrato baixo (PN). O solo destes locais foi classificado como Argissolo Vermelho (EMBRAPA, 2018) e o clima da região é do tipo "Cfa", subtropical úmido com precipitação média anual de $1.769 \mathrm{~mm}$ e temperatura média anual de $19^{\circ} \mathrm{C}$, segundo a classificação de Köppen (MORENO, 1961). No período de condução do estudo foram registradas as temperaturas e precipitação pluviométrica apresentadas na Figura 1.

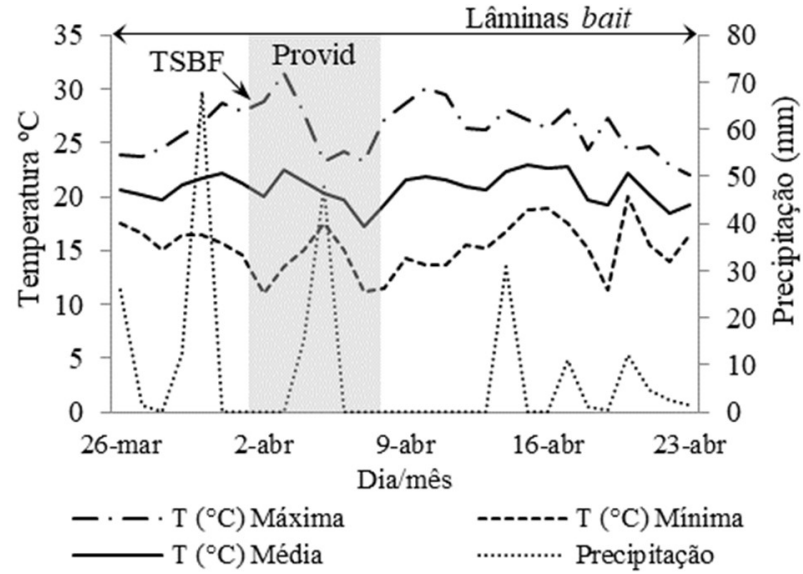

Figura 1. Precipitação pluviométrica, temperaturas média, mínima e máxima registradas durante a amostragem da fauna do solo. As lâminas bait permaneceram no campo no período de 26 de março a 23 de abril. A área hachurada representa o período de coletada dos organismos epiedáficos por meio do Provid. A coleta dos monólitos de solo (TSBF) foi realizada em 02 de abril. Santa Maria, RS. Fonte: INMET (2015).

Figure 1. Rainfall; maximum, minimum and mean temperature recorded during the sampling of soil fauna. The bait-lamina remained in the field from March 26th to April 23rd. The hatched area represents the sampling of epiedaphic organisms by Provid. Soil monoliths (TSBF) were collected on April 2nd. Santa Maria, RS. Fonte: INMET (2015).

Para a avaliação da diversidade da fauna epiedáfica empregou-se o método de armadilha de queda do tipo PROVID (ANTONIOLLI et al., 2006), que consiste na instalação de uma garrafa plástica com capacidade de dois litros, contendo quatro aberturas na forma de janelas (dimensões de 6 x $4 \mathrm{~cm}$ a $20 \mathrm{~cm}$ de sua base). No interior $\mathrm{da}$ garrafa foi adicionado $200 \mathrm{~mL}$ de solução de álcool 70\% $(\mathrm{v} / \mathrm{v})+3 \%(\mathrm{v} / \mathrm{v})$ glicerina. Foram instaladas cinco armadilhas em cada área de estudo, que capturaram organismos da fauna edáfica durante sete dias.

Para a amostragem da fauna hemiedáfica, utilizou-se o método do TSBF (Tropical Soil and Biology Fertility) (ANDERSON; INGRAM, 1993). Coletou-se em cada área, cinco monólitos de $25 \mathrm{~cm}$ de largura $\times 25 \mathrm{~cm}$ de comprimento $\mathrm{x} 20 \mathrm{~cm}$ de profundidade que foram dispostos em bandejas plásticas brancas para a triagem dos organismos.

Os organismos epiedáficos e hemiedáficos coletados foram armazenados em álcool $80 \%$, e posteriormente quantificados e identificados com auxílio de microscópio estereoscópio e materiais bibliográficos.

A atividade biológica do solo foi avaliada pelo método das lâminas bait (VON TÖRNE, 1990), que consiste na introdução de lâminas de plástico no solo. As lâminas apresentavam dimensões de $120 \mathrm{~mm}$ de comprimento x 6 $\mathrm{mm}$ de largura x $1 \mathrm{~mm}$ de espessura, com 16 orifícios de 1,5 $\mathrm{mm}$ de diâmetro, espaçados $5 \mathrm{~mm}$ entre si e preenchidos por substrato $(70 \mathrm{~g}$ celulose em pó $+27 \mathrm{~g}$ farinha de trigo $+3 \mathrm{~g}$ carvão ativado). Cinco conjuntos de 16 lâminas foram instalados em cada local de estudo. As lâminas permaneceram no solo pelo período de 28 dias. Posteriormente foi contabilizado o consumo do substrato, sendo considerados os substratos consumidos e não consumidos. Além das avaliações biológicas do solo, foram coletadas amostras de solo para análise físico-química e amostras de serapilheira para determinação da matéria seca (Tabela 1).

Tabela 1. Propriedades do solo na camada de $0-20 \mathrm{~cm}$ e acúmulo de serapilheira na superfície do florestamento de Eucalyptus spp. (FE), florestamento de Pinus spp. (FP), lavoura de grãos (LA), solo impactado pela construção civil (CC) e pastagem natural (PN).

Table 1. Soil properties in 0-20 cm layer and litter accumulation on the surface of afforestation of Eucalyptus spp. (FE), afforestation of Pinus spp. (FP), grain crops (LA), soil impacted by civil construction (CC) and natural grassland (PN).

\begin{tabular}{|c|c|c|c|c|c|c|}
\hline Propriedade & FE & FP & LA & $\mathrm{CC}$ & $\mathrm{PN}$ & Método \\
\hline Argila $\left(\mathrm{g} \mathrm{kg}^{-1}\right)$ & 26 & 29 & 22 & 26 & 31 & Densímetro \\
\hline $\mathrm{MO}\left(\mathrm{g} \mathrm{kg}^{-1}\right)$ & 220 & 250 & 290 & 170 & 260 & Walkley-Black \\
\hline $\mathrm{pH}$ & 4,5 & 4,2 & 4,7 & 4,5 & 4,6 & Água (1:1) \\
\hline $\left.\mathrm{C}_{(\mathrm{mg} \mathrm{dm}}{ }^{-3}\right)$ & 15,1 & 16,9 & 12,9 & 15,1 & 18,0 & Combustão seca \\
\hline $\mathrm{N}\left(\mathrm{mg} \mathrm{dm}^{-3}\right)$ & 1,3 & 2,4 & 1,1 & 1,3 & 1,5 & Kjeldahl \\
\hline $\left.\mathrm{P}_{(\mathrm{mg} \mathrm{dm}}{ }^{-3}\right)$ & 4,1 & 10,4 & 7,5 & 4,7 & 4,6 & Mehlich-1 \\
\hline $\left.\mathrm{K}_{(\mathrm{cmolc} \mathrm{dm}}{ }^{-3}\right)$ & 104 & 84 & 68 & 36 & 124 & Mehlich-1 \\
\hline $\mathrm{Ca}\left(\mathrm{cmolc} \mathrm{dm}^{-3}\right)$ & 11,8 & 2,1 & 3,6 & 1,2 & 7,1 & $\mathrm{KCl}(1 \mathrm{~mol} \mathrm{~L}-1)$ \\
\hline $\mathrm{Mg}\left(\mathrm{cmolc} \mathrm{dm}^{-3}\right)$ & 6,3 & 1,0 & 1,8 & 0,4 & 3,5 & $\mathrm{KCl}(1 \mathrm{~mol} \mathrm{~L}-1)$ \\
\hline $\mathrm{Al}_{\left(\mathrm{cmolc} \mathrm{dm}^{-3}\right)}$ & 6,7 & 4,5 & 1,5 & 1,5 & 3,5 & $\mathrm{KCl}(1 \mathrm{~mol} \mathrm{~L}-1)$ \\
\hline Ser. $\left(\mathrm{Mg} \mathrm{ha}^{-1}\right)$ & 12,9 & 30,9 & 3,4 & 1,1 & 12,4 & Secagem $65^{\circ} \mathrm{C}$ \\
\hline
\end{tabular}

MO = Matéria orgânica; Ser. = Serapilheira.

Os dados de abundância foram submetidos à análise de variância (ANOVA) e as médias comparadas pelo teste de Scott-Knott, a 5\% de probabilidade de erro, através do programa SISVAR (FERREIRA, 2011). A diversidade da fauna edáfica foi avaliada pelo Índice de Shannon $(\mathrm{H})$ (ODUM, 1983). A riqueza (S) foi expressa pelo número total de grupos taxonômicos.

\section{RESULTADOS}

$\mathrm{O}$ uso do solo modificou a abundância, riqueza e 
diversidade da fauna epiedáfica. Foram coletados 5.413 organismos de 12 grupos taxonômicos. Os organismos do grupo Collembola representaram $75 \%$ da abundância total, seguido dos coleópteros (10\%) e himenópteros (9,5\%) (Tabela 2). Os florestamentos apresentaram as maiores abundâncias de organismos epiedáficos, com destaque para Collembola. Já os usos do solo com vegetação herbácea (pastagem natural e lavoura), apresentaram as maiores abundâncias de himenópteros e coleópteros. Além disto, na lavoura também foram observadas as maiores abundâncias de ortópteros. O solo afetado pela construção civil apresentou a menor abundância e riqueza de organismos epiedáficos, diferindo estatisticamente dos demais usos em relação a riqueza $(\mathrm{p}<0,001)$ e diferindo do florestamento de Eucalyptus spp. e Pinus spp. na abundância de indivíduos ( $\mathrm{p}=$ 0,037). A dominância de Collembola resultou em menor diversidade nas áreas de florestamentos, justificado pelo menor valor do índice de Shannon, o qual foi estatisticamente maior na lavoura e na pastagem natural $(\mathrm{p}=0,044)$.

Tabela 2. Abundância, riqueza e diversidade (Índice de Shannon) de organismos epiedáficos e hemiedáficos em florestamento de Eucalyptus spp. (FE), florestamento de Pinus spp. (FP), lavoura de grãos (LA), solo impactado pela construção civil (CC) e da pastagem natural (PN).

Table 2. Abundance, richness and diversity (Shannon Index) of epiedaptic and hemiedaphic organisms in afforestation of Eucalyptus spp. (FE), afforestation of Pinus spp. (FP), grain crops (LA), soil impacted by civil construction (CC) and natural grassland (PN).

\begin{tabular}{|c|c|c|c|c|c|c|c|c|c|c|}
\hline \multirow{2}{*}{ Grupo } & FE & FP & LA & $\mathrm{CC}$ & $\mathrm{PN}$ & FE & FP & LA & $\mathrm{CC}$ & $\mathrm{PN}$ \\
\hline & \multicolumn{5}{|c|}{ Epiedáficos $\left(\mathrm{n}^{\circ}\right.$ armadilha $\left.{ }^{-1}\right)$} & \multicolumn{5}{|c|}{ Hemiedáficos $\left(\mathrm{n}^{\circ}\right.$ monólito $\left.{ }^{-1}\right)$} \\
\hline Arachnida & 1,8 ns1 & 1,6 & 11,6 & 0 & 5 & $0,8^{\mathrm{ns}}$ & 0,4 & 0,6 & 0,2 & 1,2 \\
\hline Coleoptera & $13^{\text {ns }}$ & 14,2 & 33,8 & 20 & 31,8 & $\mathrm{OB}$ & $0 \mathrm{~B}$ & $1,6 \mathrm{~A}$ & $0 \mathrm{~B}$ & $2 \mathrm{~A}$ \\
\hline Collembola & $310,8 \mathrm{a}$ & $418 \mathrm{a}$ & $72,4 \mathrm{~b}$ & $3,6 \mathrm{~b}$ & $14,4 \mathrm{~b}$ & $0^{\mathrm{ns}}$ & 0 & 0 & 0 & 0 \\
\hline Dermaptera & $1,6^{\mathrm{ns}}$ & 2,4 & 0 & 0 & 0 & $0^{\mathrm{ns}}$ & 0 & 0 & 0 & 0 \\
\hline Hemiptera & $0 \mathrm{~ns}$ & 1,2 & 1,2 & 0,2 & 1,4 & $0,6 \mathrm{~B}$ & OB & $0 \mathrm{~B}$ & $4,2 \mathrm{~A}$ & $0 \mathrm{~B}$ \\
\hline Hymenoptera & $8 \mathrm{~b}$ & $14,6 \mathrm{~b}$ & $40,2 \mathrm{a}$ & $12,6 \mathrm{~b}$ & $27,8 \mathrm{a}$ & $0,6^{\mathrm{ns}}$ & 0 & 10,8 & 0 & 28 \\
\hline Isopoda & $0^{\mathrm{ns}}$ & 0 & 0 & 0 & 0 & $4 \mathrm{~A}$ & $0 \mathrm{~B}$ & OB & OB & $0,2 \mathrm{~B}$ \\
\hline Isoptera & $0 \mathrm{~b}$ & $3 \mathrm{a}$ & $0 \mathrm{~b}$ & $0 \mathrm{~b}$ & $0,4 \mathrm{~b}$ & OB & OB & $0 \mathrm{~B}$ & $2, \mathrm{~B}$ & $80,6 \mathrm{~A}$ \\
\hline Larvas & $0,2 \mathrm{~ns}$ & 0 & 0,6 & 0 & 0,4 & OB & $0 \mathrm{~B}$ & $2 \mathrm{~A}$ & OB & $2,6 \mathrm{~A}$ \\
\hline Oligochaeta & $0^{\text {ns }}$ & 0 & 0 & 0 & 0 & $9 \mathrm{~A}$ & $1,6 \mathrm{~B}$ & $0,8 \mathrm{~B}$ & $\mathrm{OB}$ & $5,4 \mathrm{~A}$ \\
\hline Orthoptera & $0,6 \mathrm{~b}$ & $1,8 \mathrm{~b}$ & $7,8 \mathrm{a}$ & $1,2 \mathrm{~b}$ & $1 \mathrm{~b}$ & $0,2^{\mathrm{ns}}$ & 0 & 0,4 & 0 & 0,2 \\
\hline Outros $^{2,3}$ & $0,6^{\mathrm{ns}}$ & 0 & 1,4 & 0 & 0,4 & $0 \mathrm{~ns}$ & 0,2 & 0,8 & 0 & 1 \\
\hline Abundância total & $1683 a$ & $2284 a$ & $845 b$ & $188 \mathrm{~b}$ & $413 b$ & $76 \mathrm{~B}$ & $11 \mathrm{~B}$ & $85 \mathrm{~B}$ & $33 \mathrm{~B}$ & $608 \mathrm{~A}$ \\
\hline Riqueza & $8 \mathrm{a}$ & $8 \mathrm{a}$ & $10 \mathrm{a}$ & $5 b$ & $9 \mathrm{a}$ & $6 \mathrm{~B}$ & $3 \mathrm{D}$ & $8 \mathrm{~A}$ & $3 \mathrm{D}$ & $11 \mathrm{~A}$ \\
\hline Shannon & $0,53 \mathrm{c}$ & $0,61 \mathrm{c}$ & $2,10 \mathrm{a}$ & $1,54 \mathrm{~b}$ & $2,03 \mathrm{a}$ & $1,63 \mathrm{~A}$ & $1,09 \mathrm{~B}$ & $1,85 \mathrm{~A}$ & $1,09 \mathrm{~B}$ & $1,46 \mathrm{~A}$ \\
\hline
\end{tabular}

${ }^{1}$ Médias seguidas pela mesma letra minúscula (epiedáficos) e maiúscula (hemiedáficos) na linha, não diferem estatisticamente entre si pelo teste de ScottKnott $(p \leq 0,05) ;$ ns = não significativo. Média de cinco repetições.

${ }^{2}$ Os grupos epiedáficos Mollusca, Blattaria, e Lepidoptera foram agrupados em "Outros" pois registraram menos de 3 organismos por grupo.

${ }^{3}$ Os grupos hemiedáficos Diplopoda, Enchytraeidae, Chilopoda e Lepidoptera foram agrupados em "Outros” pois registraram menos de 3 organismos por grupo.

A abundância, riqueza e diversidade de organismos hemiedáficos também foi influenciada pelo uso do solo. Isoptera, Hymenoptera e Oligochaeta foram os grupos mais abundantes, respectivamente com 51, 25 e $10 \%$ da abundância total $(\mathrm{N}=813$, Tabela 2). A pastagem natural abrigou maior abundância destes organismos, em média 12 vezes maior que os demais usos, e também a maior riqueza de grupos, porém sem diferir estatisticamente da lavoura. Destaca-se que a pastagem natural apresentou elevada abundância de isópteros. Os himenópteros foram mais abundantes nos usos com vegetação herbácea (pastagem natural e lavoura). Já as minhocas (Oligochaeta) foram coletadas em maior número no florestamento de Eucalyptus spp. e na pastagem natural. O florestamento de Pinus spp. apresentou o menor valor de abundância, e diferiu da pastagem natural $(p=0,0373)$. A riqueza e a diversidade biológica também foram menores nesta área, igualmente ao observado no solo impactado pela construção civil.

A atividade biológica do solo, avaliada pelo consumo de substrato das lâminas bait, também foi modificada pelo uso do solo (Figura 2). A maior atividade foi observada nas áreas de florestamento de Eucalyptus spp., aproximando-se de $100 \%$ de consumo em todas as profundidades. Valores intermediários foram observados na lavoura, florestamento de Pinus spp. e na pastagem natural. No florestamento de Pinus spp. o consumo foi maior na superfície do solo, o que corrobora com a alta abundância de organismos epiedáficos observados neste ambiente. Nas áreas de lavoura e campo nativo o consumo aumentou em profundidade, o que também corrobora com a maior abundância de organismos hemiedáficos observados nestes ambientes. Já o solo impactado pela construção civil mostrou consumo próximo a zero em todas as profundidades, comprovando que a baixa abundância de organismos reflete na baixa atividade de consumo do substrato.

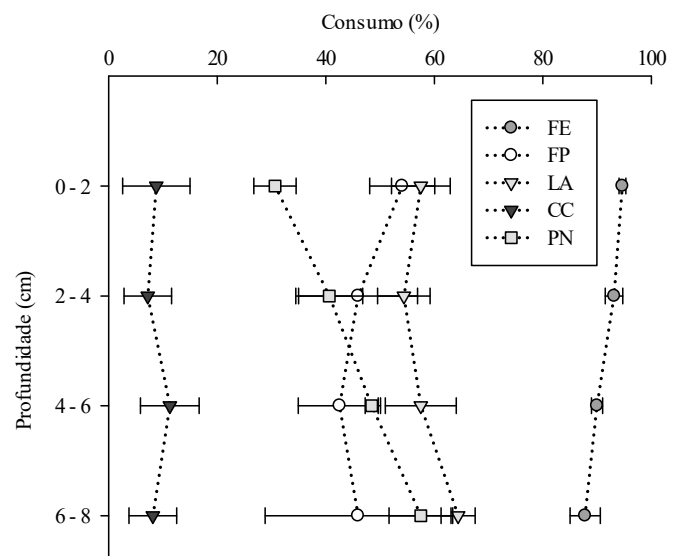

Figura 2. Atividade biológica do solo avaliada pelo consumo de substrato das lâminas bait em diferentes profundidades do solo do florestamento de Eucalyptus spp. (FE), florestamento de Pinus spp. (FP), lavoura de grãos (LA), solo impactado pela construção civil (CC) e pastagem natural (PN).

Figure 2. Soil biological activity evaluated by substrate consumption of bait-lamina at different soil depths on afforestation of Eucalyptus 
spp. (FE), afforestation of Pinus spp. (FP), grain crops (LA), soil impacted by civil construction (CC) and natural grassland (PN).

\section{DISCUSSÃO}

A fauna edáfica responde sensivelmente às alterações no ambiente, em função da abundância de alimento, formação de microclimas e aporte de recursos vegetais (BRITO et al., 2016). O grande acúmulo de serapilheira na superfície do solo do florestamento de Pinus spp. pode ter contribuído para a maior abundância de Collembola. Ao fragmentar e decompor os resíduos vegetais, a fauna do solo promove o aumento dos teores de nutrientes (CASTRO-HUERTA et al., 2015), como nitrogênio e fósforo. No florestamento de Eucalyptus spp. o elevado aporte de serapilheira também pode ter contribuído para a dominância de Collembola. Daghighi et al. (2017) sugerem que a sucessão nas comunidades de Collembola é dependente das plantas e da qualidade e quantidade de serapilheira, as quais exercem influência direta sobre importantes fatores ambientais que afetam o desenvolvimento das comunidades de Collembola, como temperatura e umidade do solo. Por outro lado, a abundância total dos organismos epiedáficos, e especialmente de Collembola, foi muito reduzida na área de ação antrópica; caracterizada pelo baixo e homogêneo aporte de serapilheira, constituída basicamente de poáceas. Neste local o menor teor de matéria orgânica e nutrientes também contribuiu para a redução da diversidade e abundância de organismos do solo. Menores quantidades de material vegetal na superfície podem explicar a redução de Collembola, que consomem esse recurso (POLLIERER; SCHEU, 2017) e indiretamente beneficiam os demais integrantes da biota do solo ao aumentar a sua superfície disponível.

$\mathrm{Na}$ lavoura e na pastagem natural coletou-se elevado número de himenópteros. As formigas desempenham importantes funções no solo, como aeração (CREPALDI et al., 2014), decomposição da matéria orgânica e ciclagem de nutrientes (BRITO et al., 2016). O alto teor de matéria orgânica no solo de lavoura está relacionado aos processos de decomposição de resíduos mediados por organismos do solo. Corroborando com os resultados encontrados no presente estudo, Silva et al. (2008) encontraram grande expressividade de insetos sociais (Formicidae) na cultura da soja cultivada sob sistema convencional e sistema integrado. Além disso, Rovedder et al. (2009) observaram elevada abundância de Hymenoptera em pastagem natural do bioma Pampa.

A maior diversidade e riqueza de organismos epiedáficos na lavoura pode ser consequência do pousio, que propiciou o crescimento de grande diversidade de espécies vegetais espontâneas, como Ipomoea spp., Cyperus spp., Solanum americanum e muitos representantes da família Poaceae. A complexidade da vegetação e a composição da serapilheira suportam comunidades mais diversas de organismos, devido ao aumento de microsítios e da disponibilidade de alimento para diferentes grupos da fauna edáfica (PEREZ et al., 2013). Contrariamente, solos intensivamente modificados pela atividade antrópica, com deposição de poluentes, habitat fragmentado com baixa diversidade vegetal e com modificações no microclima (RZESZOWSKI et al., 2017) representam condições limitantes à fauna edáfica e tendem a apresentar menores valores de riqueza e diversidade, como observado no solo impactado pela construção civil.
O ambiente da pastagem natural apresentou adequadas propriedades químicas, físicas e biológicas do solo, que propiciaram elevada abundância, riqueza e diversidade de organismos hemiedáficos. Os isópteros se destacaram com elevada abundância, corroborando com Rovedder et al. (2009) e Rosa et al. (2015). Este grupo de organismos desempenha importantes funções ecológicas em ecossistemas tropicais, atuando na movimentação do solo entre camadas e nos processos de decomposição e ciclagem de nutrientes (JOUQUET et al., 2014). Santos et al. (2008) sugerem que a alta densidade de isópteros em pastagem esteja associada à baixa fertilidade dos solos, os quais geralmente apresentam baixo percentual de matéria orgânica e alta relação $\mathrm{C} / \mathrm{N}$. No entanto, os maiores teores de argila e matéria orgânica encontrados neste estudo podem ter condicionado à alta ocorrência de isópteros, uma vez que estes organismos utilizam a argila como principal componente estrutural de seus ninhos (OBERST et al., 2016). Além disso, Rosa et al. (2015) também reportam que em pastagem nativa tais condições favorecem a construção de montículos e galerias nos ninhos.

Por outro lado, a floresta de Pinus spp. e o solo impactado pela construção civil abrigaram as menores abundâncias, riquezas e diversidades de organismos hemiedáficos. $\mathrm{Na}$ floresta de Pinus spp. registrou-se a ocorrência dos grupos Arachnida, Lepidoptera e Oligochaeta, que apresentou a maior abundância $(n=8)$. Nesta área foi encontrado o maior teor de fósforo $\left(10,4 \mathrm{mg} \mathrm{dm}^{-3}\right)$ e uma considerável relação $\mathrm{Ca} / \mathrm{Mg}(2,1)$, propriedades químicas que tem apresentado relação positiva com a presença de minhocas (ROSA et al., 2015). A baixa qualidade da serapilheira do florestamento de Pinus spp. pode ter reduzido a diversidade e riqueza dos grupos, visto que este recurso está diretamente relacionado à condições bióticas essenciais à presença da fauna edáfica, como disponibilidade de abrigo e alimento (CUNHA NETO et al., 2012)

A avaliação da atividade biológica do solo pelas lâminas bait é um método simples, de baixo investimento e que auxilia a inferir sobre o impacto do uso do solo nos organismos que integram esse sistema (KLIMEK et al., 2015). Na área da construção civil, o intenso uso do solo; o baixo teor de matéria orgânica, serapilheira e nutrientes resultou na menor atividade dos organismos do solo em todas as profundidades avaliadas. Segundo Rozen et al. (2010), a distribuição vertical da atividade biológica do solo está relacionada com a densidade da fauna, a temperatura e a umidade do solo. Logo, em áreas com baixo aporte de serapilheira, como campo nativo e lavoura, os organismos podem ter migrado em profundidades, na busca por menores temperaturas e maior umidade do solo. Por outro lado, verificou-se que na área de florestamento de Eucalyptus spp., a atividade biológica foi maior nas profundidades $0-4 \mathrm{~cm}$ possivelmente em virtude da maior quantidade de matéria orgânica (RÖMBKE et al., 2006) e da serapilheira, que proporcionam nichos e microhabitats nos primeiros centímetros do solo. A abundância pronunciada de Oligochaeta também pode explicar esse comportamento, visto que as minhocas são as principais responsáveis pela remoção do substrato em lâminas bait (RÖMBKE et al., 2006). 


\section{CONCLUSÕES}

O uso do solo modifica a diversidade, a abundância e a atividade da fauna edáfica.

Os florestamentos de Pinus spp. e Eucalyptus spp. favorecem a abundância de grupos epiedáficos.

A comunidade de organismos hemiedáficos é abundante e diversificada na pastagem natural.

A atividade biológica do solo é maior no florestamento de Eucalyptus spp. em função da qualidade e quantidade de serapilheira na superfície do solo.

O intenso uso do solo na área de construção civil reduz a abundância, riqueza e atividade biológica dos organismos do solo.

\section{REFERÊNCIAS}

ALMEIDA, D. O.; BAYER, C.; ALMEIDA, H. C. Fauna e atributos microbiológicos de um Argissolo sob sistemas de cobertura no Sul do Brasil. Pesquisa Agropecuária Brasileira, Brasília, v. 51, n. 9, p. 1140-1147, 2016. DOI: http://dx.doi.org/10.1590/s0100-204x2016000900013

ANDERSON, J. M.; INGRAM, J.S.I. Tropical Soil Biology and Fertility: a handbook of methods. 2.ed. Wallingford: CAB International, 1993. $171 \mathrm{p}$.

ANTONIOLLI, Z. I.; CONCEIÇÃO, P. C.; BÖCK, V.; PORT, O.; SILVA, D. M.; SILVA, R. F. Método alternativo para avaliar a fauna do solo. Ciência Florestal, Santa Maria, v. 16, n. 4, p. 407-417, 2006. DOI: http://dx.doi.org/10.5902/198050981922

BRITO, M. F.; TSUJIGUSHI, B. P.; OTSUBO, A. A.; SILVA, R. F.; MERCANTE, F. M. Diversidade da fauna edáfica e epigeica de invertebrados em consórcio de mandioca com adubos verdes. Pesquisa Agropecuária Brasileira, Brasília, v. 51, n. 3, p. 253-260, 2016. DOI: http://dx.doi.org/10.1590/S0100-204X2016000300007

BROWN, G. G.; NIVA, C. C.; ZAGATTO, M. R. G.; FERREIRA, S. A.; NADOLNY, H. S.; CARDOSO, G. B. X.; SANTOS, A.; MARTINEZ, G. A.; PASINI, A.; BARTZ, M. L. C.; SAUTTER, K. D.; THOMAZINI, M. J.; BARETTA, D.; SILVA, E.; ANTONIOLLI, Z. I.; DECAËNS, T.; LAVELLE, P. M.; SOUSA, J. P.; CARVALHO, F. Biodiversidade da fauna do solo e sua contribuição para os serviços ambientais. In: PARRON, L.M.; GARCIA, J. R.; OLIVEIRA, E. B.; BROWN, G. G.; PRADO, R. B. (Ed.). Serviços ambientais em sistemas agrícolas e florestais do bioma Mata Atlântica. Brasília: Embrapa, 2015. p. 113-145.

CASARIL, C. E.; DE OLIVEIRA FILHO, L. C. I.; SANTOS, J. C. P.; DA ROSA, M. G. Fauna edáfica em sistemas de produção de banana no Sul de Santa Catarina. Revista Brasileira de Ciências Agrárias, Recife, v. 14, n. 1 e5613, $2019 . \quad$ DOI: https://dx.doi.org/10.5039/agraria.v14i1a5613

CASTRO-HUERTA, R. A.; FALCO, L. B.; SANDLER, R. V.; COVIELLA, C. E. Differential contribution of soil biota groups to plant litter decomposition as mediated by soil use. PeerJ, Califórnia, v. 5, n. 3, e826, 2015. DOI: https://dx.doi.org/10.7717/peerj.826

CREPALDI, R. A.; PORTILHO, I. I. R.; SILVESTRE, R.; MERCANTE, F. M. Formigas como bioindicadores da qualidade do solo em sistema integrado lavoura-pecuária. Ciência Rural, v. 44, n. 5, p. 781-787, 2014. DOI: http://dx.doi.org/10.1590/S0103-84782014000500004
CULLINEY, T. Role of arthropods in maintaining soil fertility. Agriculture, Basel, v. 3, n. 4, p. 629-659, 2013. DOI: https://doi.org/10.3390/agriculture3040629

CUNHA NETO, F. V.; CORREIA, M. E. F.; PEREIRA, G. H. A.; PEREIRA, M. G.; LELES, P. S. S. Soil fauna as an indicator of soil quality in forest stands, pasture and secondary forest. Revista Brasileira de Ciência do Solo, Viçosa, v. 36, n. 5, p. 1407-1417, 2012. DOI: http://dx.doi.org/10.1590/S0100-06832012000500004

DAGHIGHI, E.; KOEHLER, H.; KESEL, R.; FILSER, J. Long-term succession of Collembola communities in relation to climate change and vegetation. Pedobiologia, Jena, v. 64, p. 25-38, 2017. DOI: https://dx.doi.org/10.1016/j.pedobi.2017.06.001

EMBRAPA_EMPRESA BRASILEIRA DE PESQUISA AGROPECUÁRIA. Sistema brasileiro de classificação de solos. 5.ed. Brasília, DF, 2018. 590 p.

FERREIRA, C. R.; GUEDES, J. N.; ROSSET, J. S., ANJOS, L. H. C.; PEREIRA, M. G. Diversity of the edaphic macrofauna in areas managed under no-tillage for different periods. Semina: Ciências Agrárias, Londrina, v. 40, n. 2 , p. 599-610, 2019. DOI: http://dx.doi.org/10.5433/1679-0359.2019v40n2p599

FERREIRA, D. F. Sisvar: a computer statistical analysis system. Ciência e Agrotecnologia, Lavras, v. 35, n. 6, p. 1039-1042, 2011.2 DOI: http:/ /dx.doi.org/10.1590/S1413-70542011000600001

GISIN, H. Ökologie und Lebensgemeinschaften der Collembolen im schweizerischen Exkursionsgebiet Basels. Revue Suisse de Zoologie, Gevene, v. 50, p. 131-224, 1943.

INMET_INSTITUTO NACIONAL DE METEOROLOGIA. Dados meteorológicos Estações automáticas. Santa Maria, 2015. Disponível em:

http://www.inmet.gov.br/portal/index.php?r=estacoes /estacoesAutomaticas

JOUQUET, P.; BLANCHART, E.; CAPOWIEZ, Y. Utilization of earthworms and termites for the restoration of ecosystem functioning. Applied Soil Ecology, Amsterdam, v. 73 , p. 34-40, 2014. DOI: https://dx.doi.org/10.1016/j.apsoil.2013.08.004

KLIMEK, B.; NIKLIŃSKA, M.; JAŹWA, M.; CHODAK, M.; TARASEK, A. Application of the bait-lamina method to measure the feeding activity of soil fauna in temperate forests. Polish Journal of Ecology, Warsaw, v. 63 , n. 3, p. 291-300, 2015. DOI: https://doi.org/10.3161/15052249PJE2015.63.3.011

MENTA, C. Soil fauna diversity: Function, soil degradation, biological indices, soil restoration. In: LAMEED, G. A. Biodiversity conservation and utilization in a diverse world. Gbolagade Akeem Lameed, IntechOpen, 2012. DOI: https://dx.doi.org/10.5772/51091

MOREnO, J. A. Clima do Rio Grande do Sul. Porto Alegre: Secretaria da agricultura, 1961. 42 p.

OBERST, S.; LAI, J.C.S.; EVANS, T.A. Termites utilise clay to build structural supports and so increase foraging resources. Scientific Reports, Londres, v. 8, n. 6, e20990, 2016. DOI: https://dx.doi.org/10.1038/srep20990

ODUM, E. P. Ecologia. Rio de Janeiro: Guanabara. 1983. 434 p. 
PEREZ, G.; DECAËNS, T.; DUJARDIN, G.; AKPAVINCESLAS, M.; LANGLOIS, E.; CHAUVAT, M. Response of collembolan assemblages to plant species successional gradient. Pedobiologia, Jena, v. 56, n. 4-6, p. 169-177, 2013.2013 DOI: https://doi.org/10.1016/j.pedobi.2013.04.001

POLLIERER, M. M.; SCHEU, S. Driving factors and temporal fluctuation of Collembola communities and reproductive mode across forest types and regions. Ecology and Evolution, Hoboken, v. 7, n. 12, p. 4390 4403, 2017. DOI: https://dx.doi.org/10.1002/ece3.3035

RÖMBKE, J.; HÖFER, H.; GARCIA, M. V. B.; MARTIUS, C. Feeding activities of soil organisms at four different forest sites in Central Amazonia using the bait lamina method. Journal of Tropical Ecology, Cambridge, v. 22, n. 3, p. 313-320, 2006. DOI: http://dx.doi.org/10.1017/S0266467406003166

ROSA, M. G.; FILHO, O. K.; BARTZ, M. L. C.; MAFRA, A. L.; SOUSA, J. P. A.; BARETTA, D. Macrofauna edáfica e atributos físicos e químicos em sistemas de uso do solo no planalto catarinense. Revista Brasileira de Ciência do Solo, Viçosa, v. 39, n. 6, p. 1544-1553, 2015. DOI: http://dx.doi.org/10.1590/01000683rbcs20150033

ROVEDDER, A. P. M.; ELTZ, F. L. F.; DRESCHER, M. S.; SCHENATO, R. B.; ANTONIOLLI, Z. I. Organismos edáficos como bioindicadores da recuperação de solos degradados por arenização no Bioma Pampa. Ciência Rural, Santa Maria, v. 39, n. 4, p. 1061-1068, jul. $2009 . \quad$ DOI: http://dx.doi.org/10.1590/S0103-84782009005000023
ROZEN, A.; SOBCZYK, L.; LISZKA, K.; WEINER, J. Soil faunal activity as measured by the bait-lamina test in monocultures of 14 tree species in the Siemianice common-garden experiment, Poland. Applied Soil Ecology, Amsterdam, v. 45, n. 3, p. 160-167, 2010. DOI: https://dx.doi.org/10.1016/j.apsoil.2010.03.008

RZESZOWSKI, K.; ZADROŻNY, P.; NICIA, P. The effect of soil nutrient gradients on Collembola communities inhabiting typical urban green spaces. Pedobiologia, Jena, v. 64, p. 15-24, 2017. DOI: https://dx.doi.org/10.1016/j.pedobi.2017.06.003

SANTOS, G. G.; SILVEIRA, P. M.; MARCHÃO, R. L.; THIERRY, B.; BALBINO, L. C. Macrofauna edáfica associada a plantas de cobertura em plantio direto em um Latossolo Vermelho do Cerrado. Pesquisa Agropecuária Brasileira, Brasília, v. 43, n. 1, p. 115-122, 2008. DOI: http://dx.doi.org/10.1590/S0100204X2008000100015

SILVA, J.; JUCKSCH, I.; TAVARES, R. C. Invertebrados edáficos em diferentes sistemas de manejo do cafeeiro na Zona da Mata de Minas Gerais. Revista Brasileira de Agroecologia, Rio de Janeiro, v. 7, n. 2, p. 112 125, 2012. SILVA, R. F.; AQUINO, A. M.; MERCANTE, F. M.; GUIMARÃES, M. F. Macrofauna invertebrada do solo em sistema integrado de produção agropecuária no Cerrado. Acta Scientiarum: Agronomy, Maringá, v. 30, p. 725-731, 2008.

VON TÖRNE, E. Assessing feeding activities of soil-living animals: bait-lamina-tests. Pedobiologia, Jena, v. 34, n. 2, p. 89-101, 1990. 